\title{
Preparation of two novel Schiff base polymers and their Eu (III)/Yb (III) complexes
}

Dingjun Zhang

Associate Professor, Lanzhou University of Technology, Lanzhou, China Youzhi Wu

Professor, Lanzhou University of Technology, Lanzhou, China Caixia Huo

Associate Professor, Lanzhou City University, Lanzhou, China
Ling $\mathrm{He}$

Associate Professor, Lanzhou University of Technology, Lanzhou, China Wenjiang Lu

Professor, Lanzhou University of Technology, Lanzhou, China

Zhaoxuan Feng

Graduate student, Lanzhou University of Technology, Lanzhou, China (corresponding author: fengzhaoxuan01@163.com)

A series of novel Schiff base photosensitive polymers with coordination property were synthesized through a condensation reaction. Then using the Schiff base photosensitive polymers as macromolecular ligands, a series of polymer rare-earth complexes were prepared through a coordination reaction. The structure of the polymer and their corresponding complex was performed by means of Fourier transform infrared and ultraviolet spectroscopy; the fluorescence properties of the complex were mainly investigated by fluorescence spectroscopy, and the thermal property was investigated by differential scanning calorimetry and thermogravimetric analysis. The luminescence properties of the europium (Eu) (III)/ytterbium (Yb) (III) complex in dimethylformamide solutions were investigated. The experimental results show that the complexes have fine thermal stability and all the europium (III)/ytterbium (III) complexes exhibit characteristic photoluminescence peaks in the visible region and the results indicate that the complexes are promising in the field of light-emitting materials and can be further mixed with a suitable polymer to form functional rare-earth polymers.

\section{Introduction}

In recent years, photosensitive polymers with coordination property and their rare-earth complexes have attracted significant attention due to their high thermal stability, chemical resistance, optoelectronic properties and efficient blue photoluminescent (PL) and electroluminescent (EL) properties. These functional polymers not only can coordinate with rare-earth ions but can also sensitize the fluorescence emission of rare-earth ions. ${ }^{1-6}$ In this study, some novel polymer rare-earth complexes with distinct fluorescence properties have been obtained. ${ }^{7,8}$

Aromatic Schiff base polymers (ASBPs) and their complexes are quite attractive because of their potential applications as luminescent materials. ${ }^{9-13}$ They have significant advantages over common Schiff base compounds, such as high absorption efficiency, good mechanical properties and processability. ${ }^{14-16}$ Various ASBPs containing strongly absorbing chromophores can radiate strong fluorescence and undergo easy processing. ${ }^{17-19}$ In addition, Schiff base polymer ligands with a symmetrical structure can make the system more uniform. ${ }^{20}$ Another very attractive possibility is to develop such ASBPs and their rare-earth complexes as task-specific materials. One of the important tasks to improve their application as functionalized materials is the synthesis of suitable Schiff base polymers and their rare-earth complexes. ${ }^{21-23}$

Besides intrinsic academic interest, this work assumes importance because some of these complexes have been found to display a specific optoelectronic activity. Work on Schiff base polymer rareearth complexes is relatively unknown. In this paper, two novel Schiff base photosensitive polymers with coordination property, hydrazide polymers with a salicylaldehyde terminal group, were synthesized through a condensation reaction. Their europium $(\mathrm{Eu})$ (III) $\left(\mathrm{Eu}^{3+}\right) /$ ytterbium ( $\left.\mathrm{Yb}\right)$ (III) $\left(\mathrm{Yb}^{3+}\right)$ complexes were also prepared and characterized by Fourier transform infrared (FT-IR) spectroscopy, ultraviolet (UV) spectroscopy and thermal analysis, as well as fluorescence spectroscopy.

\section{Experimental procedure}

\subsection{Materials and chemicals}

Rare-earth chlorides were prepared by dissolving the corresponding oxides $(99.9 \%$ purity) in dilute hydrochloric acid and then heating the solutions appropriately. Distilled water was used in all the experiments. The other reagents and solvents were commercially purchased and used without further treatment.

\subsection{Synthesis of europium/ytterbium(dimethyl terephthalate)(hydrazine hydrate) complex}

A hydrazide polymer with a salicylaldehyde terminal group was synthesized as follows: dimethyl terephthalate (DMT; $20 \mathrm{mmol}$ ) was added to a single flask and dissolved in absolute ethyl alcohol $(100 \mathrm{ml})$; then, hydrazine $(20 \mathrm{mmol})$ was added. After $10 \mathrm{~min}$, salicylal $(0.061 \mathrm{~g})$ was added into the solution. After being refluxed and stirred at $75^{\circ} \mathrm{C}$ for $15 \mathrm{~h}$, the Schiff base polymer was obtained. It was named as L1.

The Schiff base polymer L1 was dissolved in $50 \mathrm{ml}$ of dimethylformamide (DMF). Subsequently, europium (III) chloride hexahydrate $\left(\mathrm{EuCl}_{3} \cdot 6 \mathrm{H}_{2} \mathrm{O}\right) /$ ytterbium (III) chloride hexahydrate $\left(\mathrm{YbCl}_{3} \cdot 6 \mathrm{H}_{2} \mathrm{O}\right)$ was added. The obtained complex was precipitated 
out with distilled water after the coordination reaction was conducted for $48 \mathrm{~h}$ with stirring. Then, the complex was washed thoroughly with ethanol and distilled water, and the final product europium/ytterbium(DMT)(hydrazine hydrate) was obtained and kept in a vacuum desiccator. Moreover, the molar ratio of the ligand (calculated with carbonyl groups) to the europium (III)/ytterbium (III) ion changed (from 1:1 to 5:1) gradually in these solutions.

In this paper, the poly-DMT-co-hydrazine hydrate ligand is abbreviated as L1 and the europium/ytterbium(DMT)(hydrazine hydrate) complexes are abbreviated as C1-1:1, C1-2:1, C1-3:1, C1-4:1 and C1-5:1.

\subsection{Synthesis of complex europium(DMT)(hydrazine hydrate)(1,4-phthalaldehyde)}

DMT-hydrazine hydrate-1,4-phthalaldehyde condensates was synthesized as follows: DMT $(20 \mathrm{mmol})$ was added to a threenecked flask and dissolved in hot absolute ethyl alcohol $(50 \mathrm{ml})$. Then, hydrazine $(40 \mathrm{mmol})$ was added, and 1,4-phthalaldehyde $(20 \mathrm{mmol})$ was gradually dropped into the preceding solution (completed in $40 \mathrm{~min})$. After $10 \mathrm{~min}$, salicylal $(0.5 \mathrm{mmol})$ was added into the solution. After being refluxed and stirred at $75^{\circ} \mathrm{C}$ for $15 \mathrm{~h}$, the Schiff base was obtained. It was named as L2.

The Schiff base polymer L2 $(0.47 \mathrm{~g})$ was dissolved in $50 \mathrm{ml}$ of DMF. Subsequently, europium (III) chloride hexahydrate/ ytterbium (III) chloride hexahydrate was added. The resulting complex was precipitated out with distilled water after the coordination reaction was conducted for $48 \mathrm{~h}$ with stirring. Then, the complex was washed thoroughly with ethanol and distilled water, and the final product europium(DMT)(hydrazine hydrate) (1, 4-phthalaldehyde) was obtained and kept in a vacuum desiccator.

In this paper, the poly-DMT-1, 4-phthalaldehyde-co-hydrazine hydrate ligand is abbreviated as L2 and the europium(DMT) (hydrazine hydrate)(1,4-phthalaldehyde) complex was abbreviated as $\mathrm{C} 2$.

\subsection{Physical characterization}

FT-IR spectra were measured on a Nexus 670 FT-IR spectrometer with a potassium bromide $(\mathrm{KBr})$ flake in the wave number range $4000-400 \mathrm{~cm}^{-1}$.

Differential scanning calorimetry (DSC) and thermogravimetric analyses (TGAs) were carried out on an STA 449C TG-DSC simultaneous thermal analyzer in a nitrogen atmosphere in the $25-800^{\circ} \mathrm{C}$ range and conducted at a heating rate of $10^{\circ} \mathrm{C} / \mathrm{min}$.

UV spectra were obtained with a UV-2550 UV-visible spectrophotometer. The solutions of resultant polymers and their rare-earth complexes were prepared with DMF as the solvent.

Fluorescence excitation and emission spectra were obtained by using an RF-5301 fluorophotometer in the wavelength range 200-900 nm. The samples were dissolved in DMF with different accurate weights.

\section{Results and discussion}

As described previously, the polymer L1 (L2) was first prepared; a coordination reaction between the macromolecular ligands L1 (L2) and europium (III)/ytterbium (III) ion was carried out in a DMF solution, and then the complex C1 (C2) was obtained. The chemical process of preparing the functional polymer L1 (L2) and the complex $\mathrm{C} 1(\mathrm{C} 2)$ is expressed in Schemes 1 and 2.

\subsection{Infrared spectroscopy}

The infrared spectra of polymer L1 and its corresponding europium (III)/ytterbium (III) complexes $\mathrm{C} 1$ are compared as shown in Figure 1. The band at $3432 \mathrm{~cm}^{-1}$ was attributed to $\mathrm{N}-\mathrm{H}$ stretching vibration of the polymer (Figure 1 L1). The absorption band assigned to $\mathrm{N}-\mathrm{H}$ in the complex becomes broad

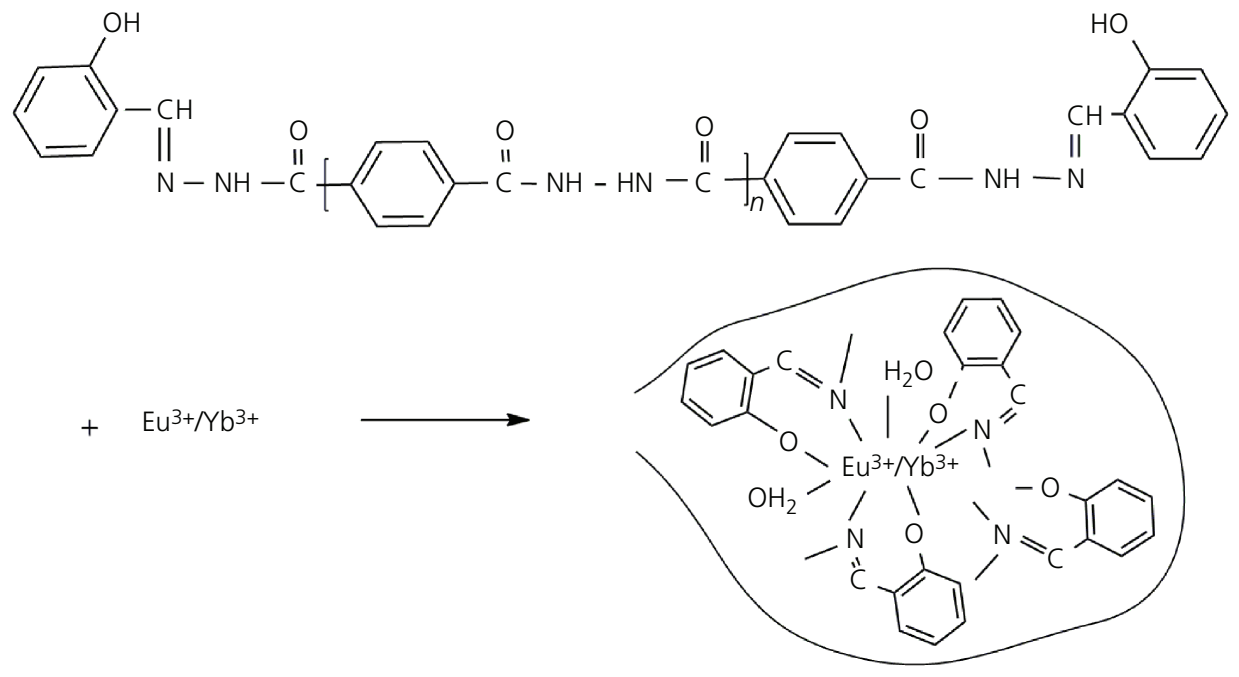

Scheme 1. Coordination reaction process of preparation of complex C1 


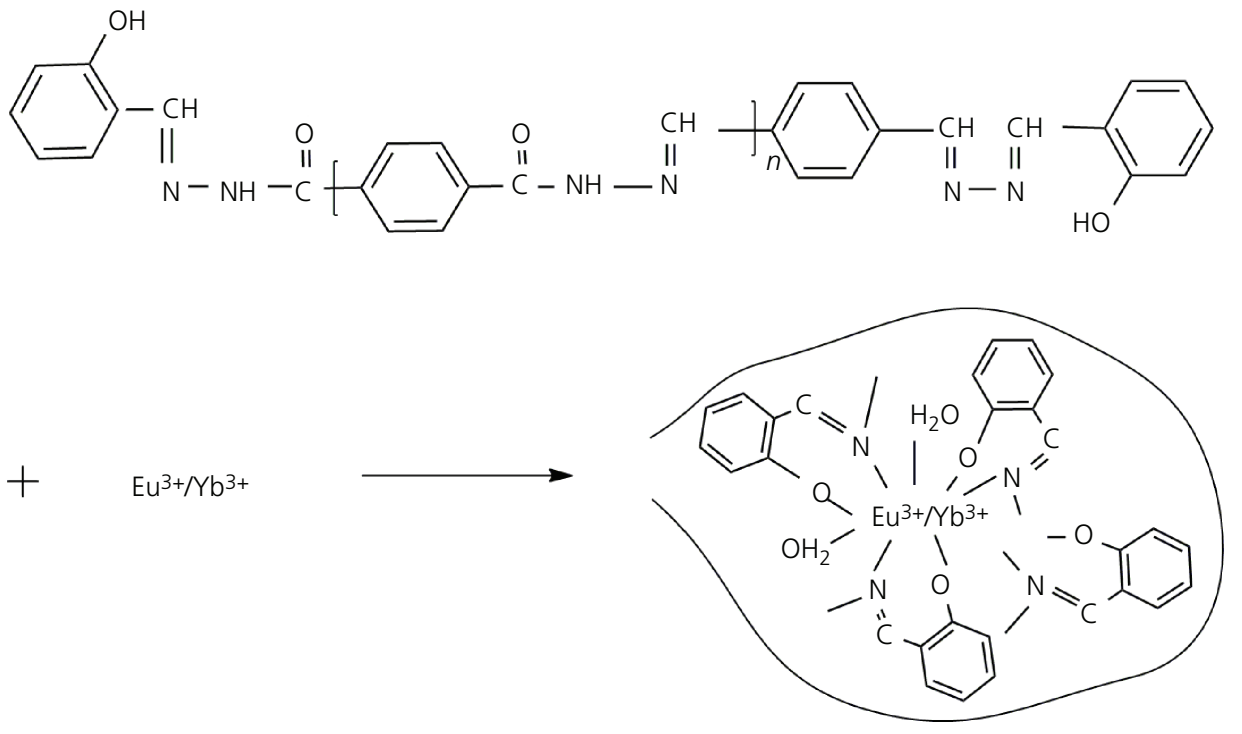

Scheme 2. Coordination reaction process of preparation of complex C2

(Figure $1 \mathrm{C} 1)$. This result is attributed to $\mathrm{M}(\mathrm{O}-\mathrm{H})$ of the water $\left(\mathrm{H}_{2} \mathrm{O}\right)$ molecule in the complex. The carbonyl stretch vibration absorption peak of the polymer appeared at $1726 \mathrm{~cm}^{-1}$ and shifted to $1721 \mathrm{~cm}^{-1}$ after being coordinated to europium (III)/ytterbium (III). These results were attributed to the macromolecular ligand coordinating with europium (III)/ytterbium (III).

The infrared spectra of polymer L2 and its corresponding europium (III)/ytterbium (III) complex $\mathrm{C} 2$ are compared as shown in Figure 2. The band at $3417 \mathrm{~cm}^{-1}$ was attributed to the $\mathrm{N}-\mathrm{H}$ stretching vibration of the polymer (Figure 2 L2). The absorption band assigned to $\mathrm{N}-\mathrm{H}$ in the complex becomes broad (Figure 2 C2). This result is attributed to $\mathrm{M}(\mathrm{O}-\mathrm{H})$ of the water molecule in the complex. The band at $1062 \mathrm{~cm}^{-1}$ is attributed to the stretching vibration absorption of $\mathrm{C}-\mathrm{N}$ (Figure $2 \mathrm{~L} 2$ ). The absorption bands at

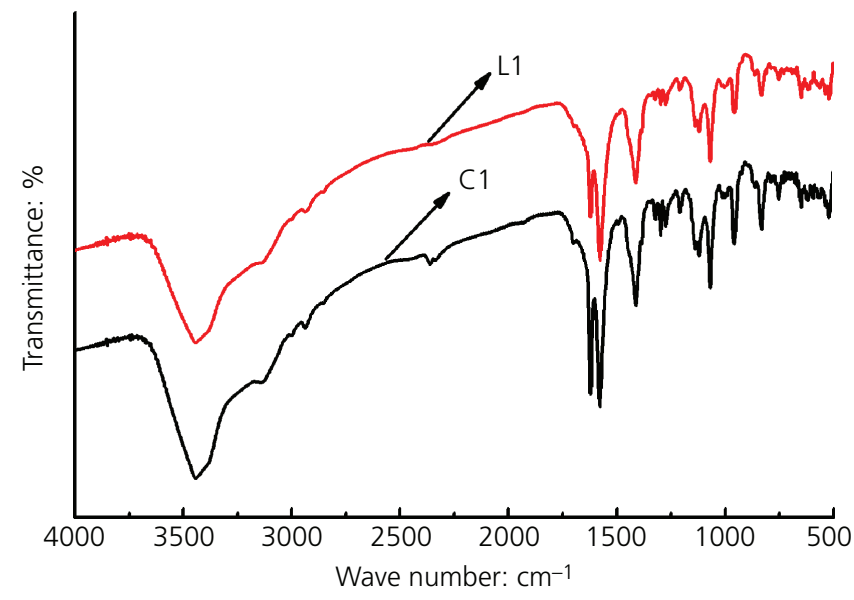

Figure 1. The infrared spectra of polymer L1 and corresponding complex C1
1721 and $1618 \mathrm{~cm}^{-1}$ are assigned to $\mathrm{C}=\mathrm{O}$ and $\mathrm{C}=\mathrm{N}$ of the polymer, respectively (Figure $2 \mathrm{~L} 2$ ). The absorption bands assigned to $\mathrm{C}=\mathrm{O}$ and $\mathrm{C}=\mathrm{N}$ in the complex shift to 1716 and $1628 \mathrm{~cm}^{-1}$, respectively. This result is attributed to the macromolecular ligand coordinating with europium (III)/ytterbium (III).

\subsection{Thermal analysis (TGA)}

Thermal analysis of polymer L1 and its corresponding complex C1 was carried out, and their TGA curves are illustrated in Figure 3. In the present study, the weight loss was measured from the ambient temperature up to $800^{\circ} \mathrm{C}$. From the TGA curves, the main degradation steps can be observed, and polymer L1 and complex $\mathrm{C} 1$ show similar trends for change in weight loss. The weight loss occurring in the temperature range $120-204^{\circ} \mathrm{C}$ is interpreted as the degradation of the ligand. At $534^{\circ} \mathrm{C}$, the

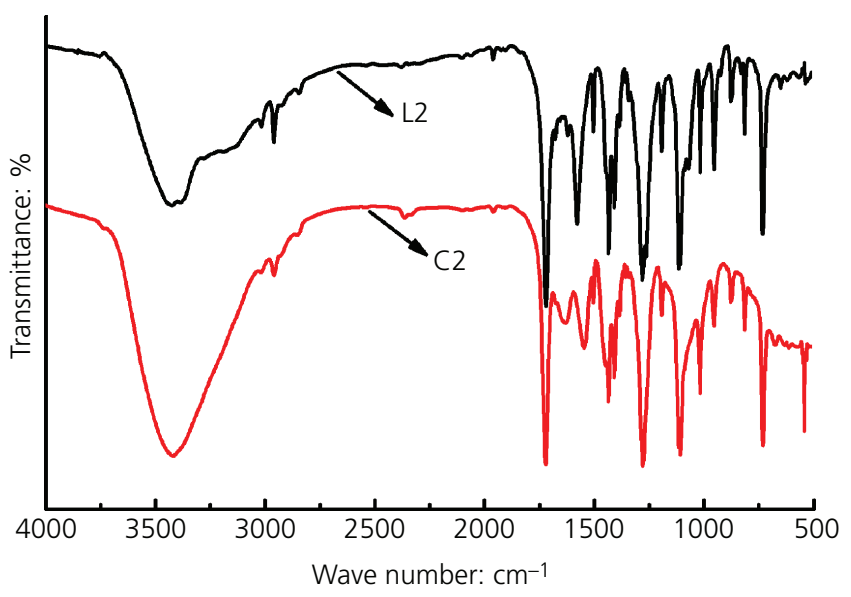

Figure 2. The infrared spectra of polymer L2 and corresponding complex C2 


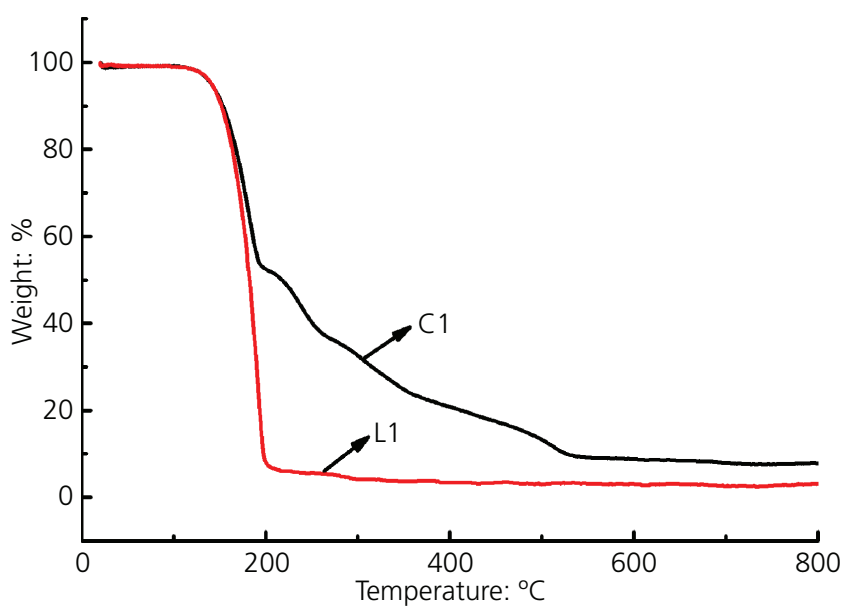

Figure 3. The thermal spectra of polymer L1 and corresponding complex C1

complex completely converts into europium (III) oxide $\left(\mathrm{Eu}_{2} \mathrm{O}_{3}\right) /$ ytterbium (III) oxide $\left(\mathrm{Yb}_{2} \mathrm{O}_{3}\right)$, and the weight becomes stable.

The thermal analysis of polymer L2 and its corresponding complex C2 was investigated (Figure 4). In the present study, the weight loss was measured from the ambient temperature up to $800^{\circ} \mathrm{C}$. From TGA curves of $\mathrm{L} 2$, it can be seen that the initial weight loss occurring in the temperature range $81-135^{\circ} \mathrm{C}$ is interpreted as loss of desorption of physically adsorbed water, residual solvent and some aromatic fragments. On further heating, there is decomposition of the organic molecular chains of $\mathrm{C} 2$ as shown in the TGA curve. At $584^{\circ} \mathrm{C}$, the complex completely converts into europium (III) oxide/ytterbium (III) oxide, and the weight becomes stable.

\subsection{UV absorption spectrometry}

Figure 5 presents the UV absorption spectra of polymer L1 and the corresponding $\mathrm{C} 1$. The following observations can be made

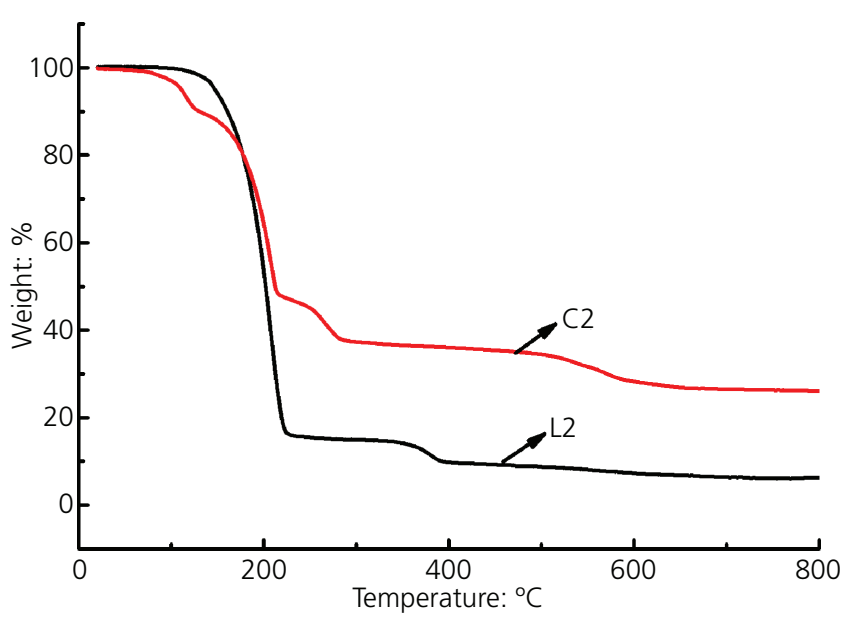

Figure 4. The thermal spectra of the polymer $L 2$ and corresponding complex C2

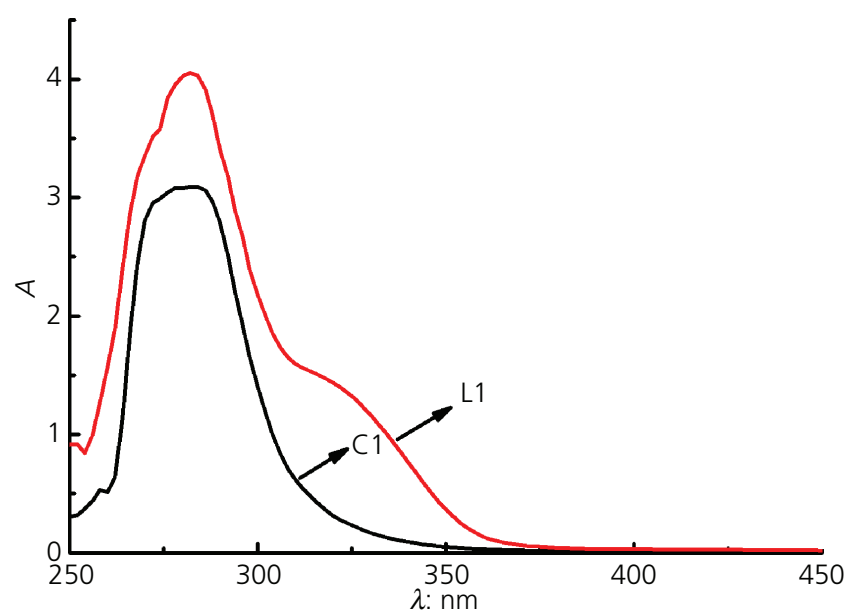

Figure 5. The UV spectra of polymer L1 and corresponding complex C1

from Figure 5: $(a)$ there is one strong absorption peak at $280 \mathrm{~nm}$, and it is ascribed to the $\pi^{*}-\pi^{*}$ electron transition of the benzene ring of the polymer. At $323 \mathrm{~nm}$, there is a little absorption peak, and it is ascribed to the $n-\pi *$ electron transition of the Schiff base ligand of polymer L1. (b) Compared with the absorption spectra of polymer L1, both the spectrum shape and the peak positions are similar to that of complex $\mathrm{C} 1$; only the characteristic absorption intensity of the absorption peak is reduced. The reduced absorption peak of the complex implies that the salicylaldehyde hydrazone ligand of polymer L1 has coordinated to europium (III)/ytterbium (III) and the complex $\mathrm{C} 1$ has been formed.

Figure 6 shows the UV absorption spectra of polymer L2 and corresponding $\mathrm{C} 2$. The following can be observed from Figure 6: (a) there is one strong absorption peak at $281 \mathrm{~nm}$, and it is

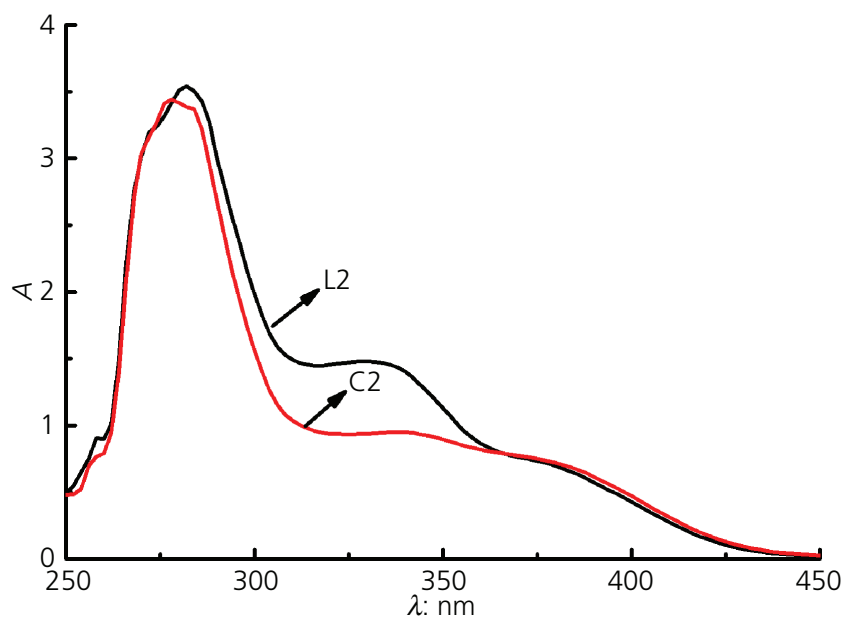

Figure 6. The UV spectra of the polymer L2 and corresponding complex C2 
ascribed to the $\pi^{*}-\pi^{*}$ electron transition of the benzene ring of the polymer. At $333 \mathrm{~nm}$, there is an absorption peak of a lower intensity, and it is ascribed to the $n-\pi^{*}$ electron transition of the Schiff base ligand of polymer L2. (b) Compared with the absorption spectra of polymer L2, the UV absorption spectra of complex $\mathrm{C} 2$ is similar to that of the polymer in shape of the spectrum and peak positions, and only the characteristic absorption intensity of the absorption peak is reduced. The reduced absorption peak of the complex implies that the salicylaldehyde hydrazone ligand of polymer L2 has coordinated to europium (III)/ytterbium (III) and the polymer rare-earth complex $\mathrm{C} 2$ has been formed.

\subsection{Fluorescence analysis}

The fluorescence excitation spectrum of $\mathrm{C} 1$ was obtained by monitoring the emission of europium (III)/ytterbium (III) ions at $489 \mathrm{~nm}$, and the optimal excitation peak was found at $463 \mathrm{~nm}$. By exciting at $463 \mathrm{~nm}$, the fluorescence emission spectrum of $\mathrm{C} 1$ in DMF solution was recorded. The result is presented in Figure 7.

From Figure 7, the following can be observed: $(a)$ the emission spectrum of $\mathrm{C} 1$ shows that the complex emits the characteristic fluorescence of the europium (III)/ytterbium (III) ion. In addition, a main emission peak at $506 \mathrm{~nm}$ is displayed, (b) When the molar ratio of the ligand salicylaldehyde hydrazone to the europium (III)/ytterbium (III) ion is equal to $5: 1$, the fluorescence emission reaches a maximum.

The fluorescence excitation spectrum of $\mathrm{C} 2$ was obtained by monitoring the emission of europium (III)/ytterbium (III) ion at $254 \mathrm{~nm}$. By exciting at $254 \mathrm{~nm}$, the fluorescence emission spectrum of $\mathrm{C} 2$ was determined. The result is presented in Figure 8.

From Figure 8, the following can be seen: the emission spectrum of $\mathrm{C} 2$ implies that the complex emits the characteristic fluorescence of the europium (III)/ytterbium (III) ion. In the

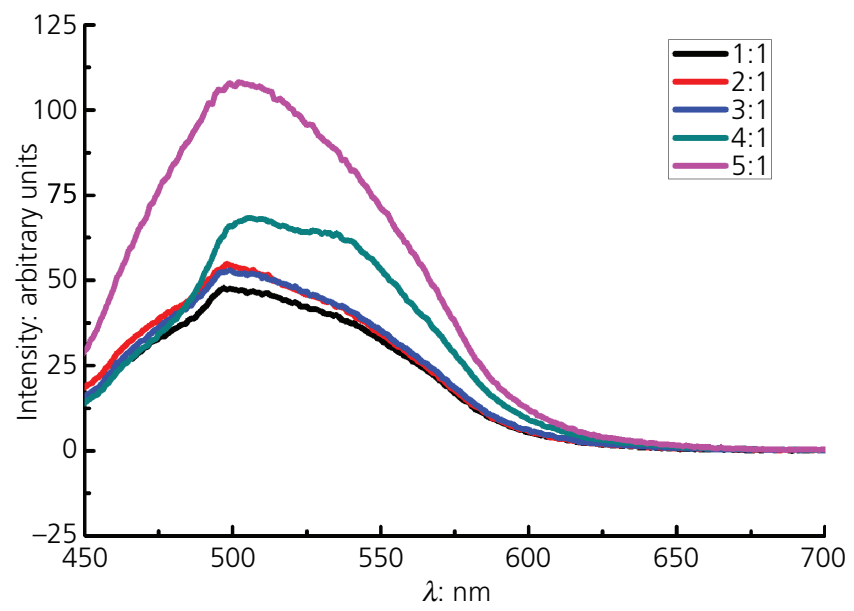

Figure 7. The fluorescence excitation spectra of complexes $C 1$

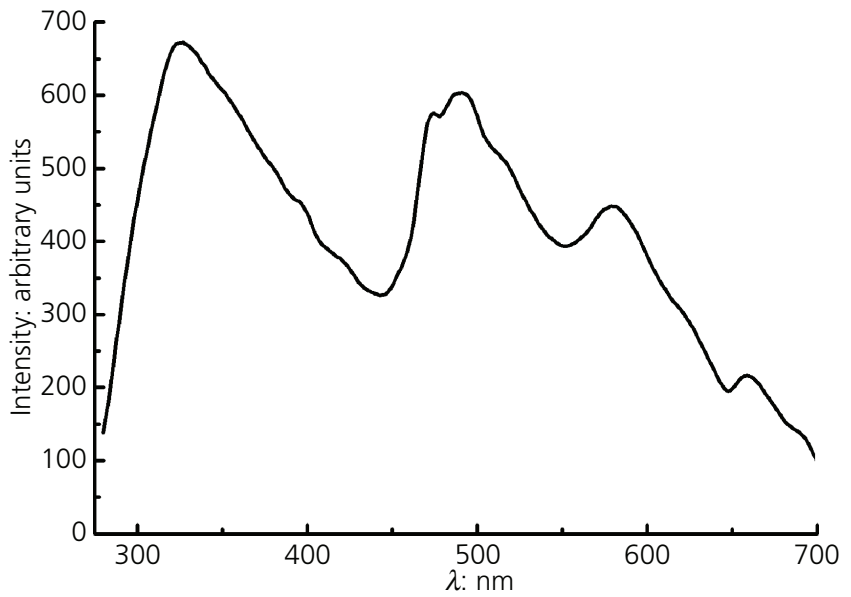

Figure 8. The fluorescence excitation spectrum of complex C2

spectrum of $\mathrm{C} 2$, three main emission peaks at 324, 486 and $579 \mathrm{~nm}$ are displayed, indicating that the Schiff base ligand of C2 can also sensitize the fluorescence emission of europium (III)/ ytterbium (III).

\section{Conclusion}

Using two Schiff base polymers prepared (L1 and L2) as functionalized macromolecular ligands, two novel Schiff base rare-earth complexes $\mathrm{C} 1$ and $\mathrm{C} 2$ were prepared through a coordination reaction, and their fluorescence emission properties were investigated in detail. The Schiff base group as a ligand and the europium (III)/ytterbium (III) ion as the central ion can form stable polymer rare-earth complexes. The aromatic Schiff base functionalized ligands L1 and L2 not only can sensitize the fluorescence emission of the central ion but also have excellent mechanical properties. It can be expected that they will have important applications in PL and EL.

\section{Acknowledgements}

The authors are grateful to the Science Foundation of State Key Laboratory of Advanced Processing and Recycling of Non-ferrous Metals (SKLAB02014009) and the Natural Science Foundation of Gansu Province of China (1310RJZA044).

\section{REFERENCES}

1. Liu D and Wang Z (2008) Novel polyaryletherketones bearing pendant carboxyl groups and their rare earth complexes. Polymer 49(23): 4960-4967.

2. Gao B, Zhang W, Zhang Z and Lei Q (2012) Preparation of polymer-rare earth complex using salicylic acid-containing polystyrene and its fluorescence emission property. Journal of Luminescence 132(8): 2005-2011.

3. Yan B and Guo M (2013) Photoluminescent hybrid alumina and titania gels linked to rare earth complexes and polymer units through coordination bonds. Inorganica Chimica Acta 399: 160-165.

4. Gao B, Qiao Z and Chen T (2014) Structure and photoluminescence property of complexes of aromatic carboxylic acid-functionalized polysulfone with $\mathrm{Eu}$ (III) and $\mathrm{Tb}$ (III). Materials Chemistry and Physics 143(3): 1119-1130. 
5. Sun Y, Yang B, Guo G, Liu Y and Zhao G (2011) Controlled formation of Sm (III) doping polymer thin films based on a new macroligand with $\beta$-diketonate. Journal of Inorganic and Organometallic Polymers and Materials 21(3): 395-399.

6. Wang DM, Cao WB and Fan J (2014) Synthesis and luminescence properties of the europium quaternary complexes nanoparticles. Science in China Series B - Chemistry 57(6): 791-796.

7. Li Y and Yang Z (2010) Rare earth complexes with 3-carbaldehyde chromone-(benzoyl) hydrazone: synthesis, characterization, DNA binding studies and antioxidant activity. Journal of Fluorescence 20(1): 329-342.

8. Li Y and Yang Z (2010) DNA-binding properties and antioxidant activity of lanthanide complexes with the Schiff base derived from 3 -carbaldehyde chromone and isonicotinylhydrazine. Journal of Coordination Chemistry 63(11): 1960-1968.

9. Singh LJ and Singh RH (2013) Synthesis and characterization of heteronuclear copper(II)-lanthanide (III) complexes of $N, N^{\prime}-1,3$ propylenebis (salicylaldiminato). International Journal of Inorganic Chemistry 2013: 281270.

10. Kausar A, Zulfiqar S, Ali L, Ishaq M and Sarwar MI (2011) Novel poly (thioureaether-imide)s derived from $4,4^{\prime}$-oxydiphenyl-bis(thiourea): probing the possibility for high-temperature applications. Polymer International 60(4): 564-570.

11. Cheetham AK, Rao CNR and Feller RK (2006) Structural diversity and chemical trends in hybrid inorganic-organic framework materials. Chemical Communications (46): 4780-4795.

12. Mitchell-Koch JT and Borovik AS (2003) Immobilization of a europium salen complex within porous organic hosts: modulation of luminescence properties in different chemical environments. Chemistry of Materials 15(18): 3490-3495.

13. Fang $Y X$, Zhao L, Wang DX and Wang MX (2012) Synthesis, structure and metal binding property of internally 1,3-arylene-bridged azacallix [6] aromatics. Journal of Organic Chemistry 77(22): 10073-10082.

14. Mahmudov KT, Kopylovich MN, Sabbatini A et al. (2014) Cooperative metal-ligand assisted E/Z isomerization and cyano activation at $\mathrm{CuII}$ and CoII complexes of arylhydrazones of active methylene nitriles. Inorganic Chemistry 53(18): 9946-9958.

15. Armelao L, Quici S, Barigelletti F et al. (2010) Design of luminescent lanthanide complexes: from molecules to highly efficient photo-emitting materials. Coordination Chemistry Reviews 254(5-6): 487-505.

16. Zhang Q, Li J, Shizu K et al. (2012) Design of efficient thermally activated delayed fluorescence materials for pure blue organic light emitting diodes. Journal of the American Chemical Society 134(36): 14706-14709.

17. Li Q, Li T and Wu J (2001) Luminescence of europium (III) and terbium (III) complexes incorporated in poly(vinyl pyrrolidone) matrix. Journal of Physical Chemistry B 105(49): 12293-12296.

18. Kannan R, He GS, Yuan L et al. (2001) Diphenylaminofluorene-based two-photon-absorbing chromophores with various $\pi$-electron acceptors. Chemistry of Materials 13(5): 1896-1904.

19. Walters KA, Ley KD, Cavalaheiro CSP et al. (2001) Photophysics of $\pi$-conjugated metal-organic oligomers: aryleneethynylenes that contain the (bpy) $\mathrm{Re}(\mathrm{CO})_{3} \mathrm{Cl}$ chromophore. Journal of the American Chemical Society 123(34): 8329-8342.

20. Hasegawa S, Horike S, Matsuda R et al. (2007) Three-dimensional porous coordination polymer functionalized with amide groups based on tridentate ligand: selective sorption and catalysis. Journal of the American Chemical Society 129(9): 2607-2614.

21. Kratsch J, Kuzdrowska M, Schmid M et al. (2013) Chiral rare earth borohydride complexes supported by amidinate ligands: synthesis, structure, and catalytic activity in the ring-opening polymerization of rac-lactide. Organometallics 32(5): 1230-1238.

22. Robinson JR, Fan X, Yadav J et al. (2014) Air- and water-tolerant rare earth guanidinium BINOLate complexes as practical precatalysts in multifunctional asymmetric catalysis. Journal of the American Chemical Society 136(22): 8034-8041.

23. Dutta S, Hung WC, Huang BH and Lin CC (2012) Recent developments in metal-catalyzed ring-opening polymerization of lactides and glycolides: preparation of polylactides, polyglycolide, and poly(lactide-co-glycolide). Advances in Polymer Science 245: 219-284.

\section{How can you contribute?}

To discuss this paper, please submit up to 500 words to the journal office at journals@ice.org.uk. Your contribution will be forwarded to the author(s) for a reply and, if considered appropriate by the editor-in-chief, it will be published as a discussion in a future issue of the journal.

ICE Science journals rely entirely on contributions from the field of materials science and engineering. Information about how to submit your paper online is available at www.icevirtuallibrary.com/page/authors, where you will also find detailed author guidelines. 